Feasibility of Proton Radiography for Mesoscale Radiography.

\author{
Graham Bench, \\ Thomas Felter, \\ Harry E. Martz \\ Arlyn Antolak
}

December 24, 2003 


\section{Disclaimer}

This document was prepared as an account of work sponsored by an agency of the United States Government. Neither the United States Government nor the University of California nor any of their employees, makes any warranty, express or mplied, or assumes any legal liability or responsibility for the accuracy, completeness, or usefulness of any information, apparatus, product, or process disclosed, or represents that its use would not infringe privately owned rights. Reference herein to any specific commercial product, process, or service by trade name, trademark, manufacturer, or otherwise, does not necessarily constitute or imply its endorsement, recommendation, or favoring by the United States Government or the University of California. The views and opinions of authors expressed herein do not necessarily state or reflect those of the United States Government or the University of California, and shall not be used for advertising or product endorsement purposes.

\section{Auspices Statement}

This work was performed under the auspices of the U.S. Department of Energy by University of California, Lawrence Livermore National Laboratory under Contract W-7405-Eng-48. 


\title{
Feasibility of Proton Radiography for Mesoscale Radiography.
}

\author{
Graham Bench (Energy and Environment Directorate, LLNL) \\ Thomas Felter (C\&MS Directorate, LLNL) \\ Harry Martz (Engineering Directorate, LLNL) \\ And \\ Arlyn Antolak (Materials \& Engineering Sciences Center, Sandia National Laboratories)
}

The power of sufficiently-energetic proton beams to penetrate high atomic number (Z) metals, together with the potential for high-dynamic-range measurements, enabled by the roughly linear energy loss mechanism in the material, makes ion beam imaging complementary to x-ray techniques and, in many cases, it is superior. Specifically, x-ray imaging is poor in an object that contains both low- and high$\mathrm{Z}$ materials. This is because the energetic $\mathrm{x}$-rays required to penetrate high $-\mathrm{Z}$ material(s) interact weakly with the low $-Z$ materials and therefore provide poor image contrast. Protons, on the other hand, are less sensitive to $Z$; thus they penetrate the high- $Z$ material, yet are sufficiently influenced by the low $-Z$ material as to provide useful contrast and information. Each proton "measures" the total electronic density of material that it traverses by its gradual and continuous energy loss as it passes through the object. Measuring the energy loss of a proton beam that has traveled through the target provides information about the line integral of the areal electron density in the material in a single measurement. Repeating this measurement across the target thus provides an electron-density map of the target; reconstructing multiple maps can lead to full 3-D tomographic renderings.

The use of proton beams as an imaging probe with micron-scale spatial resolution in spatially extended $(\mathrm{mm})$ targets can be hindered by positional and energy blurring known as straggling. This blurring is caused by the beam's strong interactions with the electrical charge distribution of the material through which it travels. Lateral straggling of the proton trajectories affects the spatial resolution and is a function of beam energy and target material. It was not until the mid 1990's that the problem of image degradation caused by straggling began to be addressed at LLNL though image reconstruction algorithms that corrected for the effect of beam spatial broadening. However, a major limitation of such work is that lateral straggling in many target materials has been poorly characterized for a variety of proton energies.

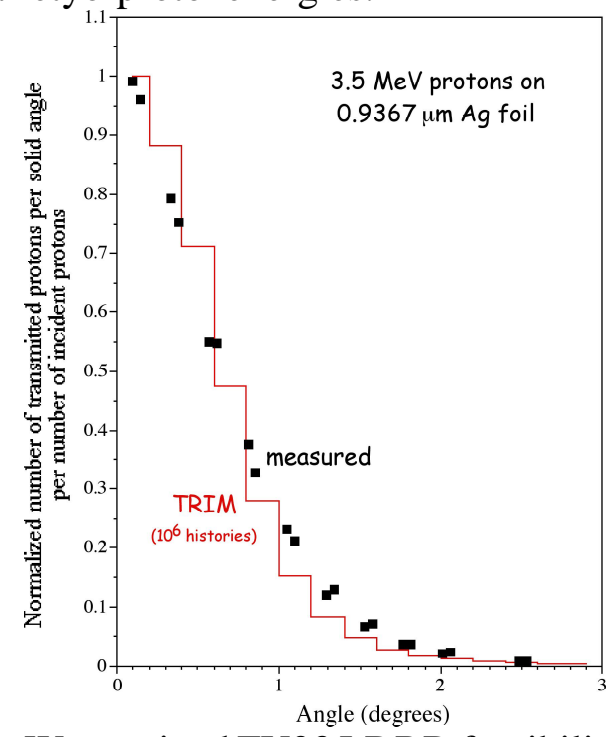

Figure 1: Measured and predicted angular straggling of $3.5 \mathrm{MeV}$ protons after passing through a 0.94 um thick silver foil.

We received FY03 LDRD feasibility funding (03-FS-028) to concentrate on experimentally characterizing the lateral and energy straggling of $\mathrm{MeV}$ protons. The 2-D straggling data have been used to extend our ion-beam modeling capabilities. As an example, Figure 1 shows the measured angular straggling of $3.5 \mathrm{MeV}$ protons after passing through a 0.94 um thick Silver foil and the corresponding computed distribution obtained using a the program Transport and Range of Ions in 
Matter (TRIM) that we modified to better model small angle scattering. The experimental and computed data have Full Width Half maximum (FWHM) values that agree within 10\%, which is adequate for our characterization/modeling requirements.

The 2-D straggling data are being incorporated into existing tomography simulation models in order to improve and predict the characterization of samples in three dimensions. The data also suggest that we could typically expect to be able to resolve micron-scale features within a variety of mesoscale objects given an operational high-energy (10's of $\mathrm{MeV}$ ) proton microbeam facility. In principle, the new models should be able to produce accurate 3-D tomographic simulation results needed for planning and evaluating experiments with pertinent material combinations and geometries. From the validated codes it should be possible to model whether proton imaging can provide the required characterization for mesoscale objects, such as high energy density physics (HEDP) targets, fuel cells or biological samples.

The success of the feasibility study strongly suggests that the next phase of our effort should be to obtain 3-D experimental tomographic data to provide the necessary validation and assessment of our updated tomographic modeling and simulation codes. This requires that both simulations be performed and experimental data be collected for well-defined test objects. Collection of the experimental data will require the high-energy ion microprobe in B190 to be refurbished so that it can collect proton tomography data with beams of energy up to $20 \mathrm{MeV}$. With this capability we could demonstrate the capability of proton tomography by performing computer simulations and proton imaging on actual targets of interest to HEDP and inertial confinement fusion (ICF). The experimental data would provide the full validation of the updated simulation capability.

While HEDP is the most relevant LLNL mesoscale characterization challenge, a high-energy proton tomographic capability would benefit a broad range of applications. For example, LLNL has an active research program to develop micro-fuel cells for personal power generation. Proton tomography would provide nondestructive three-dimensional information about the quality of bonding between various material layers and the uniformity of these layers, which presently requires destructive sectioning for examination. Proton tomography would also be able to non-destructively characterize micron sized meteorites and interplanetary dust captured in aerogels in relation to NASA's Stardust program. The ability to locate in three dimensions and quantitatively determine the density distribution of captured particles in the aerogel (both non-destructively and with fine spatial resolution) before further analysis by other techniques would provide a powerful extension to this characterization program. 